\title{
Establishment and molecular characterization of human dermal mesenchymal-like stem cells derived from human scalp biopsy of androgenetic alopecia patient ${ }^{* \#}$
}

\author{
Pravin Potdar $^{\dagger}$, Kavitha S. Kumar \\ Department of Molecular Medicine \& Biology, Jaslok Hospital \& Research Centre, Mumbai, India; \\ ${ }^{\dagger}$ Corresponding Author: ppotdar@jaslokhospital.net, ppravin012@gmail.com
}

Received 29 November 2012; revised 31 December 2012; accepted 20 January 2013

Copyright (C) 2013 Pravin Potdar, Kavitha S. Kumar. This is an open access article distributed under the Creative Commons Attribution License, which permits unrestricted use, distribution, and reproduction in any medium, provided the original work is properly cited.

\begin{abstract}
Development of Dermal cell line has great scope in the field of skin related diseases and regenerative medicine. Alopecia leads to a skin disorder causing balding and its mechanism is not yet understood. In the present study, we have developed and characterized a heterogeneous population of human dermal mesenchymal-like stem cell line from scalp biopsy of androgenetic alopecia patient with a view to isolate cells from the bulge region of the hair follicle. Our study showed that the dermal cells isolated from dermis of skin showed epithelial-like cells expressing CD34 and Keratin 18, which are characteristic of bulge hair follicle cells. These cells also expressed mesenchymal phenotypes and pluripotency markers such as Oct4, Nanog and SOX $_{2}$. These cells were designated as "Human Dermal Mesenchymal-like Stem Cells (hDMSC s)". To confirm their epithelial phenotypes, we have grown these cells at low serum concentration and it was observed that $3 \%$ serum concentration provided optimum conditions for their growth and maintenance of characteristics. The hDMSCs cells are presently at passage 10 . This study reports the establishment of human dermal mesenchymal-like cell line from the dermis of Alopecia patient, which may be used as an in vitro model system to study the

\footnotetext{
*This project is financially supported through the grant for project no 491, A/C 27814, by Research Society of Jaslok Hospital \& Research Centre, Mumbai, India.

\# The authors declare that they have no conflict of interests.

${ }^{\dagger}$ Corresponding author.
}

mechanism of Alopecia and other related skin disorders.

Keywords: Dermal Cells; Alopecia; Mesenchymal Stem Cells; Keratin 18; CD34; CD105

\section{INTRODUCTION}

The stem cell discovery and research has brought about a revolution to the field of science and technology. It has built a horizon for new ventures in tissue engineering and regenerative medicine [1,2]. Enormous methods have been put forth to isolate stem cells from various tissues which opened doors to the idea of selfcell repair and has emerged as a new technology for therapeutic and clinical applications [3-5].

Androgenetic Alopecia (AGA) is a common hair disorder, which is hereditary and androgen-dependent process, seen in genetically predisposed male and female [6]. Studies have suggested that the mechanism and origin of AGA in females is not yet clear when compared to the males. The mechanism of AGA in males is genetic and depends on the androgen receptor polymorphism, which leads to an increase in the 5-alpha reductase activity that brings about an increase in the production of dihydrotestosterone. The increase in the activity of 5-alpha reductase is said to trigger an inhibition of cell proliferation in dermal papilla and local production of vascular endothelial growth factor (VEGF). In case of women, it is suggested that AGA is triggered due to an autoimmune disorder which still needs to be investigated [6-8]. Oral finasteride, which acts as a competitive inhibitor of 5-alpha type-2 reductase and topical application of minodixil solution that stimulates the production of VEGF in cul- 
tured dermal papilla cells, are the only treatment available for AGA [8].

Recent studies have suggested that skin-derived stem cells would have potential contributions in studies related to dermatology and dermatopathology such as the skin aging, wound healing disorders and skin neoplasms; and also in the field of tissue repair, regenerative medicine, gene and cell-based therapies [9-11]. Development of different types of skin cell lines under in vitro conditions can be achieved using different components supplemented in the growth media which would give rise to different types of skin cells [12]. Transformation of human dermal papilla cell line with human telomerase reverse transcriptase gene (hTERT) induced with Simian Virus 40T (SV40T) has been carried out to establish an immortalized dermal papilla cell line, which would be useful for studies on hair and related disorders. Dermal marker study performed on these cells shows that they maintain certain dermal papilla proteins and also suggests that these cells were capable of exhibiting signaling pathways similar to that of dermal papilla cells [13].

CD34 ${ }^{+}$MSC-like cells may be suggested to be present in the human adult dermis and it is observed that the perivascular region in the dermis was rich in $\mathrm{CD} 34^{+}$cells which were capable of differentiating into mesenchymal lineages [14]. CD34 acts as a specific marker for the epithelial keratinocytes present in the hair follicle bulge and have suggested that these cells with characteristic progenitor marker could be used for tissue engineering, gene therapy and to study carcinogen target cells [15]. A study was performed on bald and non-bald scalp of AGA individuals to evaluate the presence of hair follicle stem and progenitor cells. It was observed that Keratin 15 expression was maintained in both the samples which related to their stem cell property but observed a decrease in the expression of CD200 and CD34 which posses the progenitor phenotype [16]. Another study suggested that human dermis can derive specific cell population due to its mesenchymal differential potential as these cells expressed various MSC markers such as CD73, CD90, CD 105 and also CD271, SSEA-4 [17].

The serum concentration in the growth media plays a critical role in development and differentiation of various cell types under in vitro conditions. Studies have shown that epithelial cells grow better in serum free media as compared to media containing serum as it was found that the activity of transforming growth factor-beta (TGFbeta) could differentiate epithelial cells and reduces its growth rate [18]. The effect of serum free and serum containing media on fetal and adult dermal fibroblasts have been studied and results suggest that the presence or absence of serum in both cell types did not affect the cell growth of fibroblast [19]. However, it has been shown that serum containing TGF- $\beta$ inhibit the growth of epithelial cells [18] and therefore serum free medium is used for better growth of epithelial-like cells in culture.

The present study, aimed at developing and characterizing a human dermal cell line from an androgenetic alopecia patient to understand the mechanism of alopecia and related disorder. We characterized these cells by using morphological and molecular stem cell markers to confirm the cell phenotypes. Development of Dermal skin cell line from human could be important as an in vitro model system for in-depth studies on the mechanism of skin diseases at molecular level and in the current situation dermal skin cells can be useful for regenerative therapies and for bone and cardiac related disorders.

\section{MATERIALS AND METHODS}

\subsection{Scalp Biopsy Collection and Processing for Development of Human Dermal Cell Line}

Fresh scalp biopsy from a patient (33/F) suffering from Androgenetic Alopecia was obtained in sterile $1 \times$ Phosphate Buffer Saline (PBS) (Himedia, India) with prior consent of the patient as per the guidelines of the ethical committee at Jaslok Hospital and Research Centre, Mumbai, India. The tissue explant was sent to the stem cell laboratory for further processing. This explant was washed several times with sterile $1 \times$ PBS containing $1 \%$ Penstrep (Himedia, India). The explant was dissected so as to separate the epidermal layer from the dermal layer. The dermal layer was further processed. The tissue was minced well and digested with $0.25 \%$ Trypsin-EDTA (Himedia, India) at $37^{\circ} \mathrm{C}$ for half an hour in the $\mathrm{CO}_{2}$ incubator. The explant was then placed onto $65 \mathrm{~mm}$ Nunc culture dish which were scratched so as to support the adherence of the tissue on to the petri dish. The dishes were fed with freshly prepared complete growth medium (Dulbecco's Modified Eagle's Medium, Himedia, India) that was supplemented with $10 \%$ FBS (Fetal Bovine Serum, GIBCO), $1 \%$ Penicillin - Streptomycin, $1 \mu \mathrm{l} / \mathrm{ml}$ Insulin (Sigma, USA), $2 \mu \mathrm{l} / \mathrm{ml} \cdot \mathrm{L}$-Glutamine (Himedia, India). The dishes were incubated at $37^{\circ} \mathrm{C}$ in the $\mathrm{CO}_{2}$ incubator with $5 \% \mathrm{CO}_{2}$. Regular monitoring of these dishes was done to check for outgrowth of cells from the tissue explant using the phase contrast microscope. Within 3 to 4 days, outgrowth of cells from the explant was observed and the cells adhered to the culture dish without any feeder layer or substratum. Every alternate day, the cultures were fed with growth medium and these cells achieved confluence within two weeks. Each time before the transfer of cells for an increase in passage, these cells were given an initial partial trypsinization using $0.25 \%$ Trypsin-EDTA for 1minute to remove the fibroblasts cells from the pool of other cells. Once these cells were removed, the rest of the cells were kept in trypsin for another 2 minutes, neutralized with fresh growth media and then transferred to $50 \mathrm{~mm}$ Nunc culture flasks for 
expansion of these cultured cells. This process was repeated when the cells were confluent. A heterogeneous population of skin cells was obtained after several weeks of culturing. Presently, the cell line is at passage 10. Excessive cells were cryopreserved at $-86^{\circ} \mathrm{C}$ for further characterization by cellular and molecular markers $[3,20]$.

\subsection{Effect of Various Concentrations of Fetal Bovine Serum on the Dermal Cell Line}

The Epithelial Dermal cells such as the keratinocytes which grow better in serum free or low serum containing medium and therefore we have studied the effect of different concentrations of serum containing growth medium on this cell line. The sub-confluent dermal cell cultures growing in T50 flask were taken for this study. 2 $\times 10^{4}$ cells were transferred to each five $65 \mathrm{~mm}$ Nunc petri dishes and were fed with normal growth media and incubated in the $\mathrm{CO}_{2}$ incubator for $24 \mathrm{hr}$. Next day, these cells were washed with $1 \times$ PBS and fed with growth media containing 10\%, 5\%, 3\%, 1\% Fetal Bovine Serum (FBS) and serum free media. The cells were monitored after an interval of $2 \mathrm{hr}, 4 \mathrm{hr}$ and $8 \mathrm{hr}$, then after every 24 hr for 8 days to check for any morphological changes under phase contrast microscope. The cells before and after treatment were photographed before they were sacrificed for RNA extraction after 8 days in culture to study the expression of molecular markers.

\subsection{Extraction of RNA from Dermal Skin Biopsy (in Vivo) and Dermal Cell Line (in Vitro)}

Total RNA was extracted using Trizol method (Invitrogen, Carlsbad, USA) from the dermal layer of the skin biopsy obtained from the Alopecia patient (in vivo study), dermal cell line (in vitro study) and dermal cells treated with different concentration of serum. The RNA was reverse transcribed to complementary DNA (cDNA) using Applied Biosystems high capacity cDNA kit (Applied Biosystems, USA) and used for RT/PCR of various molecular markers such as CD34, Keratin 18, IL-6, CD $105, \mathrm{CD} 73, \mathrm{CD} 13$, Oct4, Nanog, $\mathrm{SOX}_{2}$, and $\beta$-Actin (internal control) as described in previous studies [3-5,20]. The amplified PCR products were run on 2\% Agarose gel electrophoresis and observed under the gel documentation system (Cell Biosciences) and were photographed.

\section{RESULTS}

\subsection{Development and Phase Contrast Microscopy of Human Dermal Cell Line from Scalp Biopsy of Androgenetic Alopecia Patient}

Isolation of human dermal cells from scalp biopsy of
Androgenetic Alopecia patient was previously described in the materials and methods. Regular monitoring of the culture dishes was done every day and within 3-4 days of incubation, outgrowth of cells was observed. Phase contrast microscopy of the development of dermal cell line was shown in Figures 1(a)-(d). Figure 1(a) shows the hDMSCs cells spindle-in shape with large nucleus and nucleoli with scanty granular cytoplasm. The cells showed high rate of proliferation with doubling time of $18 \mathrm{hr}$. Figure 1(b) shows the confluent culture of the dermal cell line at passage 10 showing elongated cells with large nucleus having 1 - 2 nucleoli, which grew as monolayer cultures. Growth of Epithelial-like cells is shown in Figure 1(c). Figure 1(d) shows the formation of pluripotency clones in the dermal cells.

\subsection{Molecular Characterization of Human Dermal Skin Biopsy (in Vivo) and Human Dermal Cell Line (in Vitro)}

In our present study, we have studied 9 molecular stem cell markers for the characterization of Human Dermal skin biopsy (in vivo) and isolated dermal cells (in vitro) by RT/PCR technology.

\subsection{Expression of CD34, Keratin 18, IL-6 and CD105 in Human Dermal Skin Biopsy and the Dermal Cell Line}

Figure 2 shows the expression of CD34, Keratin 18, IL-6 and CD105 molecular markers in human dermal skin biopsy and the dermal cell line. It was observed that CD34 expression was completely down regulated in Alopecia skin biopsy due to the diseased condition. How-

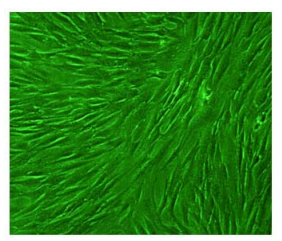

(a)

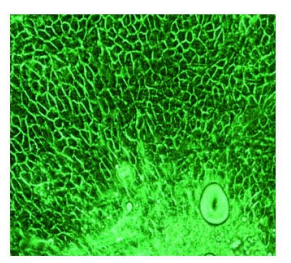

(c)

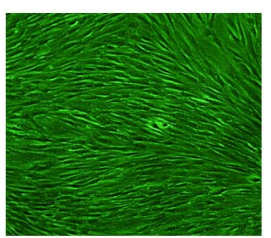

(b)

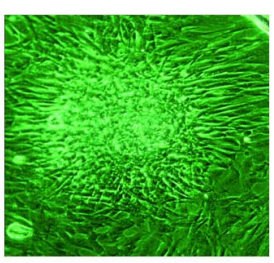

(d)
Figure 1. (a) Shows hDMSCs cells derived from skin biopsy of Androgenetic Alopecia patient outgrows from the explant within 3 4 days in incubation; (b) Shows an increase in number of cells after two weeks in culture; (c) Shows cell line grown as monolayer cells in culture at passage 10; (d) Shows the formation of pluripotency clone in hDPSCs cells. 


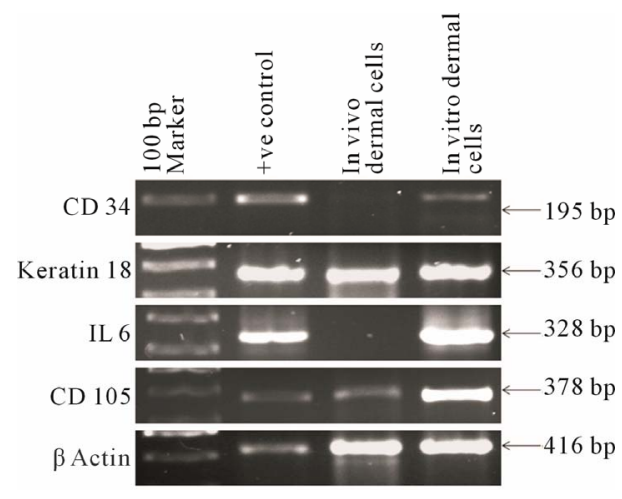

Figure 2. Shows the expression of CD34, Keratin 18, IL-6 and CD105 in Human Dermal Skin Biopsy and hDMSCs cell line.

ever epidermal cells prominently expressed CD34 indicating that under culture conditions, the cells present in Alopecia skin biopsy revert back to normal phenotype as shown in Figure 2. The differentiation marker Keratin 18 was expressed in dermal skin biopsy as well as in the dermal cell line indicating that the cells maintain their epithelial phenotypes (keratinocyte-like property) as it is observed under in vivo condition (Figure 2). It was also observed that IL-6 was down regulated in the Alopecia skin biopsy due to the diseased condition as reported. However, expression of IL-6 was significantly increased in the dermal cell line, which is similar as found in normal human skin (Figure 2). The expression of CD105 suggested the presence of Mesenchymal phenotype in dermal skin biopsy and dermal cell line as shown in Figure 2. Based on the observation from the phase contrast microscopy and the gene expression pattern, these cells were designated as Human Dermal Mesenchymal-like Stem cell line (hDMSCs).

\subsection{Effect of Serum Concentration on the MorphoLogical Features of hDMSCs Cell Line}

Prominent expression of Keratin 18 was seen in hDMSCs cells, which indicate the presence of epithelial phenotypes suggestive of resemblance to the keratinocytes present in skin. Epithelial cells grow better in serum free or low serum containing media. In the present study, we have tested the cell growth potential of hDMSCs cell line by treatment with various concentrations of serum.

\subsection{Expression of Specific Stem Cell Markers in hDMSCs Cells Treated with Various Concentrations of FBS}

\subsubsection{Expression of Skin Specific Markers in hDMSCs Cell Line (CD34 and Keratin 18)}

Our previous results have shown that hDMSCs cells expressed CD34 and Keratin 18 marker, which resemble the expression similar to normal skin cells. Figure 3 shows the expression of CD34 and Keratin 18 gene expression in hDMSCs cells treated with various concentrations of FBS. It was clearly observed that hDMSCs cells expressed significant amount of CD34 and Keratin 18 at 3\% concentration of FBS (Figure 3 ) than at other concentration.

\subsubsection{Expression of Mesenchymal Markers in hDMSCs Cell Line (CD105, CD73 and CD13)}

We have already reported that hDMSCs cells expressed CD105, which is one of the mesenchymal phenotype markers. We have added two more specific mesenchymal markers i.e. CD73 and CD13 to confirm these findings. It was observed from the study that hDMSCs cells showed significant expression of all three genes at $3 \%$ concentration of serum when compared to cells grown at other concentration as shown in Figure 4, which indicates that hDMSCs cells possess mesenchymal cell-like property.

\subsubsection{Expression of Pluripotency Markers in hDMSCs Cell Line (Oct4, Nanog and $\mathrm{SOX}_{2}$ )}

Maintenance of pluripotency is important for the continued growth of these cells with an increase in the number of passages. In the present study, we have studied pluripotency genes Oct4, Nanog and $\mathrm{SOX}_{2}$ at different concentration of FBS. On comparison of hDMSCs cells

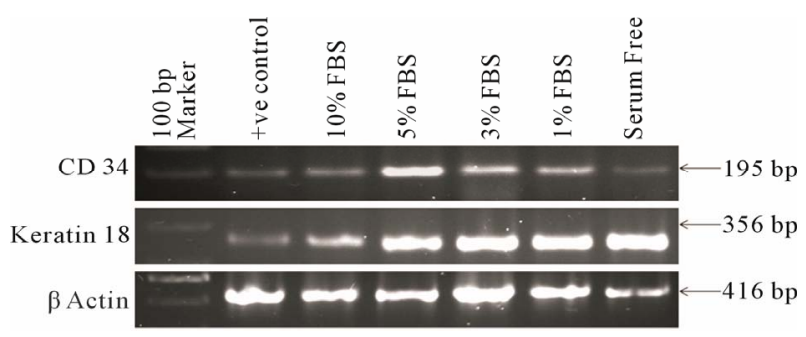

Figure 3. Shows the expression of Skin specific markers (CD34 and Keratin 18) in hDMSCs cell line.

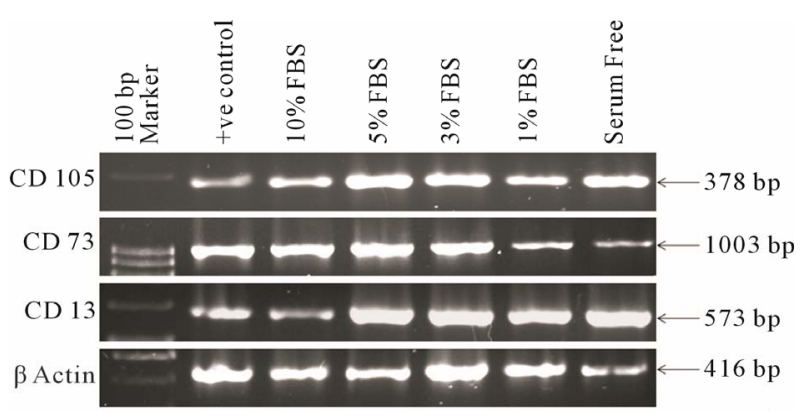

Figure 4. Shows the expression of Mesenchymal markers (CD105, CD73 and CD13) in hDMSCs cell line. 
at $3 \%$ FBS with other concentration of serum it was observed that all three pluripotency genes were significantly expressed in these cells indicating presence of pluripotent phenotypes in this cell line (Figure 5).

\section{DISCUSSION}

Cell lines derived from skin have gained increasing importance, as they are easy to culture and have the ability to differentiate into varied cell types $[10,11]$. Androgenetic Alopecia is identified as a hereditary disorder which causes baldness in men as well as the females but the mechanism behind this was chalked out only in case of males whereas in females it is yet to be understood [6-8]. Having an in vitro model system would help to understand such mechanisms. In this study, we developed and characterized human scalp biopsy derived dermal cell line from the bulge region in the dermis of an AGA patient, which was designated as the hDMSCs cell line. These cells showed high rate of proliferation and morphologically they were elongated and spindle-shaped cells. The present study mainly dealt to understand the mechanism of these cells at the molecular level under diseased condition, using stem cell specific molecular markers. Previous studies have stated that the expression of CD34 and IL-6 is absent in person suffering from alopecia where as present under normal conditions [16, 21]. We observed that CD34 and IL-6 expression had completely down regulated in the dermal skin biopsy whereas, the hDMSCs cell line expressed these genes. This may indicate that the cells derived from the dermal skin biopsy of the Alopecia patient reverts back to normalcy under culture conditions. It can be suggested that these cells could be useful to understand the mechanism of Alopecia at the genetic level. As a heterogeneous population of skin-derived cells was obtained, it was also observed that Keratin 18 was expressed suggesting the presence of keratinocytes. The mesenchymal stem cell marker CD105 showed increased expression on the hDMSCs cells whereas mild expression was observed in the dermal skin biopsy cells. This is suggestive of the derived cell line to exhibit mesenchymal property, which

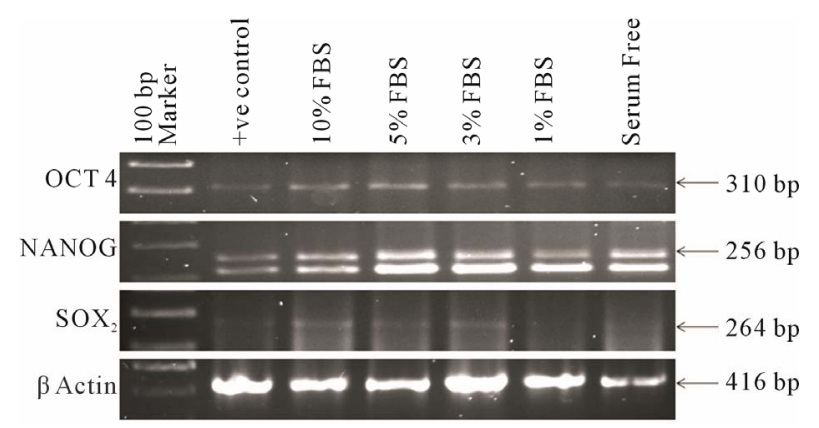

Figure 5. Shows the expression of Pluripotency markers (Oct 4, Nanog and $\mathrm{SOX}_{2}$ ) in hDMSCs cell line. makes it more desirable to be used for clinical and therapeutic process.

Studies have suggested role of serum in the in vitro growth and development of epithelial cell line. It is observed that TGF- $\beta$ present in the serum could inhibit the growth of these cells and cause differentiation in these cells and reduced its growth rate [18]. To study this mechanism, we treated the hDMSCs cells with different concentration of serum to study its effects on the growth and molecular characterization of these cells. We observed that at $3 \%$ FBS concentration, the hDMSCs cell line prominently expressed the skin specific markers (CD34 and Keratin 18), the mesenchymal stem cell markers (CD105, CD13 and CD73) and the pluripotency markers (Oct4, Nanog and $\mathrm{SOX}_{2}$ ). Lowering the serum concentration does not affect the growth rate and functioning of the hDMSCs cell line which increases its potential to be used as an in vitro model system for understanding the mechanism of action of various skin related disorder and also for its clinical applications.

Overall our study describes the establishment of the hDMSCs cell line resembling the normal skin cells. Molecular characterization helps us to further confirm maintenance of hDMSCs phenotypes under culture conditions. This is the first study to establish normal human dermal mesenchymal stem cell line from dermal skin biopsy of AGA patient, which could be useful for application of dermal skin cells in the field of dermatology, regenerative medicine and tissue engineering.

\section{ACKNOWLEDGEMENTS}

The authors would like to thank and acknowledge the management of Jaslok Hospital and Research Centre, Mumbai for funding and Dr. Afsha Topal for providing the tissue sample for this project.

\section{REFERENCES}

[1] Badylak, S.F. and Nerem, R.M. (2010) Progress in tissue engineering and regenerative medicine. Proceedings of the National Academy of Sciences USA, 107, 3285-3286. doi:10.1073/pnas.1000256107

[2] Trounson, A. (2009) New perspectives in human stem cell therapeutic research. BioMedCentral Medicine, 7, 29.

[3] Potdar, P.D. and Sutar, J.P. (2010) Establishment and molecular characterization of mesenchymal stem cells derived from human visceral and subcutaneous adipose tissue. Journal of Stem cells and Regenerative Medicine, 6, 26-45.

[4] Potdar, P.D. and D'Souza, S.B. (2011) Isolation of Oct4 ${ }^{+}$, Nanog $^{+}$and $\mathrm{SOX}_{2}^{-}$mesenchymal cells from peripheral blood of a diabetes mellitus patient. Human Cell, 24, 51-55. doi:10.1007/s13577-011-0011-6

[5] Potdar, P.D. and Chaugule, S. (2011) Establishment and molecular characterization of breast cancer mesenchymal stem cell line derived from human non-metastasis breast 
cancer tumor. Stem Cell Discovery, 1, 21-28. doi:10.4236/scd.2011.12003

[6] Jamin, C. (2002) Androgenetic alopecia. Ann Dermatol Venereol, 129, 801-803.

[7] Price, V.H. (2003) Androgenetic alopecia in women. Journal of Investigative Dermatology Symposium Proceedings, 8, 24-27.

doi:10.1046/j.1523-1747.2003.12168.x

[8] Trüeb, R.M. (2002) Molecular mechanisms of androgennetic alopecia. Experimental Gerontology, 37, 981-990. doi:10.1016/S0531-5565(02)00093-1

[9] Lau, K., Paus, R., Tiede, S., Day, P. and Bayat, A. (2011). Exploring the role of stem cells in cutaneous wound healing. Experimental Dermatology, 18, 921-933. doi:10.1111/j.1600-0625.2009.00942.x

[10] Sellheyer, K. and Krahl, D. (2010) Skin mesenchymal stem cells: Prospects for clinical dermatology. Journal of the American Academy of Dermatology, 63, 859-865. doi:10.1016/j.jaad.2009.09.022

[11] Zouboulis, C.C., Adjaye, J., Akamatsu, H., Moe-Behren, G. and Niemann, C. (2008). Human skin stem cells and ageing process. Experimental Gerontology, 43, 986-997. doi:10.1016/j.exger.2008.09.001

[12] Abdel-Naser, M.B., Abdallah. M., Larangeira de Almeida Jr., H. and Wollina, U. (2005) Human skin cell culture and its impact on dermatology. Egyptian Dermatology Online Journal, 1, 1-25.

[13] Shin, S.H., Park, S.Y., Kim, M.K., Kim, J.C. and Sung, Y.K. (2011) Establishment and characterization of an immortalized human dermal papilla cell line. Biochemistry and Molecular Biology Reports, 44, 512-516.

[14] Yamanishi, H., Fujiwara, S. and Soma, T. (2012). Perivascular localization of dermal stem cells in human scalp. Experimental Dermatology, 21, 78-80. doi:10.1111/j.1600-0625.2011.01407.x

[15] Trempus, C.S., Morris, R.J., Bortner, C.D., Cotsarelis, G., Faircloth, R.S., Reece, J.M. and Tennant, R. (2002). En- richment for living murine keratinocytes from the hair follicle bulge with the cell surface marker CD34. Journal of Investigative Dermatology, 120, 501-511. doi:10.1046/j.1523-1747.2003.12088.x

[16] Garza, L.A., Yang, C., Zhao, T., Blatt, H.B., Lee, M., He, H., Stanton, D.C., Carrasco, L., Spiegel, J.H., Tobias, J.W. and Cotsarelis, G. (2011) Bald scalp in men with androgenetic alopecia retains hair follicle stem cells but lacks CD200-rich and CD34-positive hair follicle progenitor cells. The Journal of Clinical Investigation, 121, 613-622. doi:10.1172/JCI44478

[17] Vaculik, C., Schuster, C., Bauer, W., Iram, N., Pfisterer, K., Kramer, G., Reinisch, A., Strunk, D. and Elbe-Bürger, A. (2011) Human dermis harbors distinct mesenchymal stromal cell subsets. Journal of Investigative Dermatology, 132, 563-574. doi:10.1038/jid.2011.355

[18] Ilio, K.Y., Sensibar, J.A. and Lee, C. (1995) TGF-beta1, TGF-alpha and EGF on cell proliferation and cell death in rat ventral prostatic epithelial cells in culture. Journal of Andrology, 16, 482-490.

[19] Brink, H.E., Stalling, S.S. and Nicoll S.B. (2005). Influence of serum on adult and fetal sermal fibroblast migration, adhesion, and collagen expression. In Vitro Cellular \& Developmental Biology-Animal, 41, 252-257. doi:10.1290/0503023R.1

[20] Potdar, P.D. and Subedi, R.P. (2011) Defining molecular phenotypes of mesenchymal and hematopoietic stem cells derived from peripheral blood of acute lymphocytic leukemia patients for regenerative stem cell therapy. Journal of Stem cells and Regenerative Medicine, 7, 29-40.

[21] Kirnbauer, R., Kock, A., Schwarz, T., Urbanski, A., Krutmann, J., Borth, W., Damm, D., Shipley, G., Ansel, J.C. and Luger, T.A. (1989). IFN-beta 2, B cell differentiation factor 2 , or hybridoma growth factor (IL-6) is expressed and released by human epidermal cells and epidermoid carcinoma cell lines. The Journal of Immunology, 142, 1922-1928. 